\title{
Sensitive and Simple Detection of Glucose Based on Single Plasmonic Nanorod
}

\author{
Gang Xu,* Yuhua ZHU, ** and Jie PANG**广 \\ *Pharmaceutical Research Center and School of Chemistry and Chemical Engineering, Southeast University, \\ Nanjing 211189, China \\ **School of Chemistry \& Chemical Engineering, Nanjing University, 163 Xianlin Road, Nanjing 210023, China
}

\begin{abstract}
A simple and sensitive method to determinate glucose content was developed based on single plasmonic nanoparticles by conventional dark-field microscopy (DFM). An enzyme-responsive plasmonic Ag/Au bilayer of rods was designed and prepared. Their localized surface plasmon resonance (LSPR) could be tailored by the enzyme reaction of glucose oxidase (GOx) by finely tuning the morphology and plasmonic optical response of the hybrid nanostructure. It was found that the plasmon resonance scattering (PRS) spectra peak $\left(\lambda_{\max }\right)$ shifted to longer wavelengths under enzyme reactions, and the degree of the shift were proportional to the content of glucose. This approach is convenient to study the local concentration of glucose in real time.
\end{abstract}

Keywords Single plasmonic nanoparticles, glucose, $\mathrm{Ag} / \mathrm{Au}$ bilayer rods

(Received July 19, 2016; Accepted September 28, 2016; Published February 10, 2017)

\section{Introduction}

Noble metallic nanoparticles have attracted significant attention for their unique tunable size, shape and composition as well as their interesting catalytic, electronic, and plasmonic optical properties. $^{1-4}$ The optical properties of metal nanoparticles are determined by localized surface plasmon resonance (LSPR), which is influenced by many aspects, such as the size, shape, composition and the surrounding medium. ${ }^{5-8}$ Among nanoparticles with various shapes, an anisotropic Au nanorod (NR) has attracted considerable attention in the past few years because of their high sensitivity to the local refractive indices of the surrounding medium. ${ }^{9-11}$

Recently, bio-inspired enzyme-responsive plasmonic metal nanoparticles have become attractive since hybrid nanoparticles (NPs) have potential applications in biosensing and bioelectronics when their LSPR bands shift in response to biorecognition events or enzyme-catalyzed reactions. ${ }^{12-18}$ Most of these studies on biosensing applications have been done in solution using the UV-Vis or the surface plasmon resonance (SPR) spectrum, which washes out the analysis and tracking of individual nanoparticles. Besides, these nanomaterials are easy to aggregate in solution and hard to prepare. Therefore, new methods need to be developed to detect biomolecules. Darkfield microscopy (DFM), because of its real-time optical sensing and high sensitivity, has been used to directly observe chemical reactions by studying single plasmonic NPs. ${ }^{19-21}$

Herein, we report on an interesting hybrid enzyme-responsive $\mathrm{Au} / \mathrm{Ag}$ bimetallic nanorod (NR) system for in situ sensitive optical glucose sensing and real-time monitoring the activity of enzymes. The enzyme reaction was monitored at the single-

$\dagger$ To whom correspondence should be addressed.

E-mail: pangjie@nju.edu.cn nanoparticle level using a real-time color imaging and plasmon resonance scattering (PRS) spectra shift, which could be observed by a charge-coupled device (CCD) camera and a spectrometer, as depicted in Scheme 1.

The principle of this experiment is that the surface-modified enzyme can oxide the glucose to generate hydrogen peroxide in the presence of oxygen, which can dissolve silver to regulate the thickness of the silver coating around gold nanorods. ${ }^{15,22-24}$ Using above-mentioned principle we can tune the LSPR of $\mathrm{Au} / \mathrm{Ag}$ NRs by the enzyme reaction of glucose oxidase (GOx) through fine tuning the morphology and plasmonic optical response of the hybrid nanostructure. The reactions can be described as follows:

$$
\begin{aligned}
& \text { GOx-glucose }+\mathrm{O}_{2} \longrightarrow \text { D-gluconic acid }+\mathrm{H}_{2} \mathrm{O}_{2}, \\
& 2 \mathrm{Ag}+\mathrm{H}_{2} \mathrm{O}_{2} \longrightarrow 2 \mathrm{Ag}^{+}+2 \mathrm{OH}^{-} .
\end{aligned}
$$

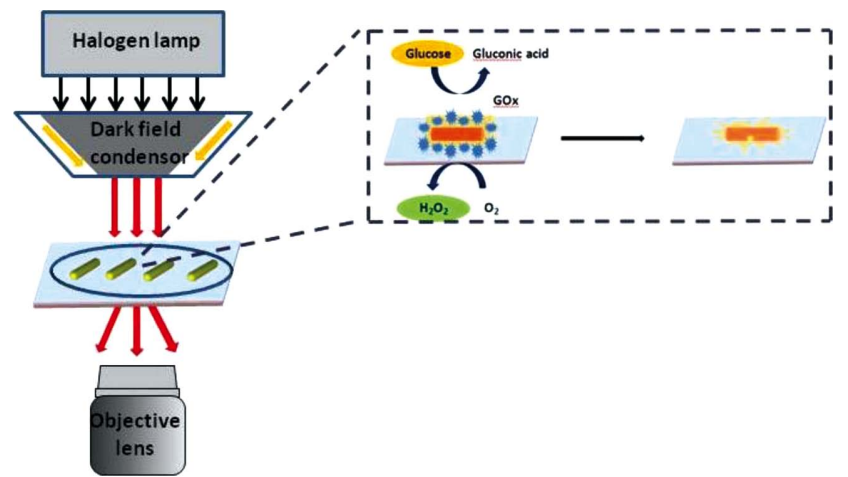

Scheme 1 Preparation of the enzyme-responsive hybrid Au/AgGOx NRs and the mechanism of glucose sensing. 


\section{Experimental}

\section{Reagents and chemicals}

All reagents were of analytical grade and used without further purification. All solutions were prepared with Milli-Q water from a Millipore system and quantified using UV-Vis absorption spectroscopy. Milli-Q water with a resistivity of $18.2 \mathrm{M} \Omega$ was used in all preparations.

\section{Apparatus}

The morphologies of the samples were characterized by scanning electron microscopy (SEM; EDAX-4800) and transmission electron microscopy (TEM; Japan JEOL-2010). Light-scattering spectra were recorded using a Cary Eclipse fluorescence spectrophotometer (Agilent Technologies, USA). Dark-field images were acquired on a Nikon inverted microscope Eclipse Ti-U equipped with a color charge coupled device (CCD; S45, Canon, Japan). The scattering spectra of single nanoparticles were recorded using an SP2556 spectrograph equipped with a 512B_excelon electron multiplying charge coupled device (Princeton Instruments, USA) and mounted on a Nikon microscope. Glass slides were cleaned under a PSDUV4 ozone system (Novascan Technologies) before use. All tests were carried out at room temperature.

In order to measure the PRS spectrum, NRs were first immobilized on a piece of glass slip by physical adsorption. Before using, the slide was washed with ethanol and distilled water thoroughly, and was then irradiated under an Ultra-Violet/ Ozone cleaner for $1 \mathrm{~h}$. Then, a piece of polydimethylsiloxane (PDMS) with a 6-mm hole was covered on the glass. The hole on the PDMS then was used as a container for an NRs solution for later glucose measurements. Next, a drop of diluted $\mathrm{Au} / \mathrm{Ag}$ NRs solution $(\sim 0.01 \mathrm{nM})$ was added in the PDMS hole and allowed to contact with the glass surface for $30 \mathrm{~min}$ to obtain a reasonable surface density of single nanoparticles. The glass was then washed with pure water three times in order to remove free NRs in the solution. During the following measurement, a drop of $10 \mathrm{mM}$ PBS $(200 \mu \mathrm{L})$ always covered the glass. A darkfield imaging mode of the microscope was first used to obtain images of single NRs. The PRS spectra of the single NR were recorded by turning the light pathway to a spectrometer connected on the microscope. An electron multiplying chargecoupled device (EMCCD) was used to record the spectra.

\section{Results and Discussion}

The Au/Ag bimetallic NRs used as sensing platforms were first synthesized as reported. ${ }^{25}$ Figure 1 shows SEM images of the precursor Au NRs (Fig. 1a), TEM images of hybrid Au/Ag NRs (Fig. 1b) and the UV absorption of $\mathrm{Au} / \mathrm{Ag}$ solution and $\mathrm{Au} / \mathrm{Ag}$ immobilized on the glass slide (Fig. 2e). All of the above characterizations indicate that the Au/Ag NRs had a uniform size. The average diameter and length of the $\mathrm{Au} / \mathrm{Ag}$ NRs were $12 \pm 1$ and $51 \pm 3 \mathrm{~nm}$, with a shell thickness of $2-3 \mathrm{~nm}$. The $\mathrm{Au} / \mathrm{Ag}-\mathrm{GOx}$ NR complex was then formed by electrostatic adsorption of the negatively charged GOx protein $(\mathrm{PI}=4.2)$ onto the positively charged and CTAB-coated NRs surface at $\mathrm{pH}$ 7.4. During the process, an excess amount of GOx was used to reach the monolayer saturate adsorption on the NRs surface; then, any excess GOx was removed before the addition of glucose.

Scattering images and spectra of GOx-modified Au/Ag NRs were obtained using DFM coupled with a spectrograph. As
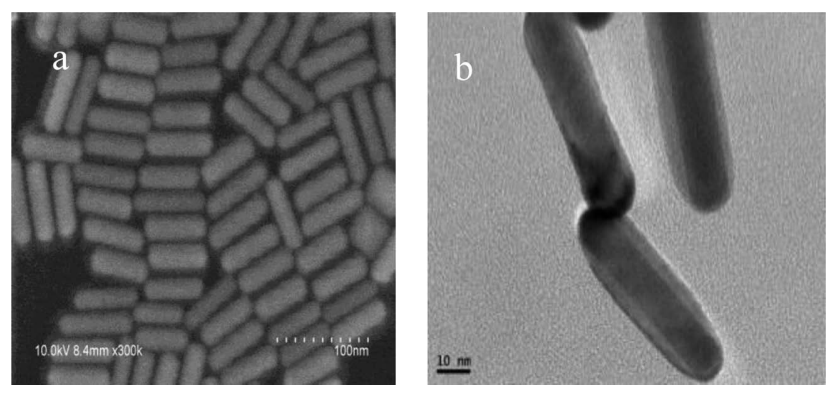

Fig. 1 (a) SEM image of the Au NRs, (b) TEM image of Au/Ag bimetallic NRs.

shown in Fig. 2f, a small spectral redshift of about $2 \mathrm{~nm}$ occured after GOx was electrostaticly attached onto the Au/Ag NR surfaces. The scattering peaks of the complex Au/Ag-GOx NRs for longitudinal plasmon resonance were mostly around $620 \mathrm{~nm}$ (Fig. 2b) with a red color (Fig. 2a). However, upon the addition of glucose, a redshift of the scattering spectrum took place, and change of the imaging color and brightness in the dark field were also observed (Figs. 2c-2d). The statistics of 25 spectra of colored NRs shows that most of their peak positions are around $620 \mathrm{~nm}$, and that the stability and reliability of each NR are acceptable. The control experiment shows that the maximum peak of every single NR, itself, will not shift for hours. The above data provided a basis for designing the nanosensor using a single $\mathrm{Au} / \mathrm{Ag} \mathrm{NR}$. Figure $2 \mathrm{f}$ shows typical PRS spectral changes of the $\mathrm{Au} / \mathrm{Ag}-\mathrm{GOx} \mathrm{NRs}$, before and after incubation with glucose for $60 \mathrm{~min}$. From the scattering spectra, it is clear that the max of the scattering spectrum of the Au/Ag-GOx NRs exhibits a peak redshift of about $12 \mathrm{~nm}$. Meanwhile, the applicability of this sensing system was investigated. The changes in the scattering shift of NRs were determined after incubation with various concentrations of glucose $\left(10^{-5}-\right.$ $\left.10^{-3} \mathrm{M}\right)$. Dots with similar color and brightness were chosen in the CCD so as to discuss the relationship between the $\lambda_{\max }$ shifts and the glucose concentrations, because the NRs' sizes and their delicate surface environments were not the same. As shown in Fig. 3a, the higher was the glucose concentration, the bigger did the shift of the max of the PRS spectrum become. In addition, the shift was found to be linear with the glucose concentration from $0.5 \times 10^{-5}$ to $10^{-4} \mathrm{M}$ (Fig. 3d). The detection limit (LOD) was $0.5 \mu \mathrm{M}$, under the concentration in which the scattering spectrum shifts by around $4 \mathrm{~nm}$, which is lower than those obtained using reported fluorescence detection methods. ${ }^{26-29}$ However when the glucose concentration was above $0.5 \mathrm{mM}$, the shift of spectrum peaks reached a saturation value of $13 \mathrm{~nm}$, since the Ag outer Au Rods all became dissolved. The control experiment showed that no detectable spectra shift of the $\mathrm{Au} / \mathrm{Ag}$ NRs (GOx-free) occurred after incubation with $3 \times 10^{-4} \mathrm{M}$ of glucose for $60 \mathrm{~min}$. A quantitative analysis of the scattering $\lambda_{\max }$ statistical distribution of the nanoparticles before and after the reaction was also performed (Figs. $2 b$ and $2 d$ ). Because of the shell change outside the Au NR, a shift of the spectrum was recorded before and after etching. The successful PRS spectra $\lambda_{\max }$ response of single $\mathrm{Au} / \mathrm{Ag}$ NRs exhibited a distinct peakshift behavior toward longer wavelengths, the average shift was about $15 \mathrm{~nm}$.

In order to better know the sensing progress, we investigated the time-dependent evolution of the scattering spectra of the asprepared $\mathrm{Au} / \mathrm{Ag}-\mathrm{GOx}$ NRs incubated with $3 \times 10^{-4} \mathrm{M}$ glucose solutions. The data in Fig. $3 \mathrm{~d}$ indicate that the sensing progress 

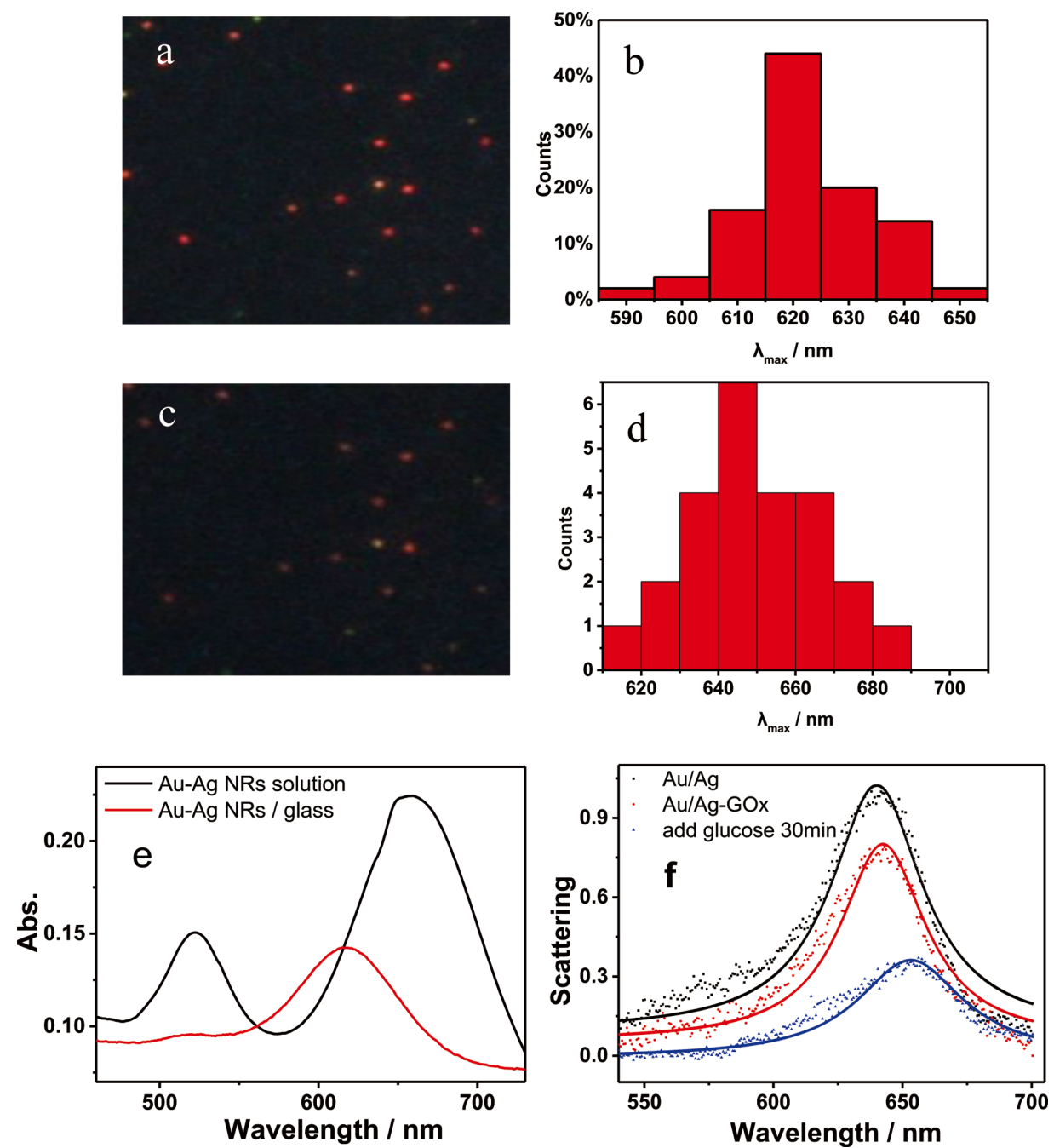

Fig. 2 Typical DFM images of single GOx-modified Au/Ag NRs before (a) and after (c) the reaction. Statistical distribution of scattering $\lambda_{\max }$ for single Au/Ag-GOx before (b) and after (d) the reaction; the measurements were carried out on a minimum of 30 independent particles per sample. (e) UV-Vis spectra of the colloidal Au/Ag NRs (black) and Au/Ag NRs immobilized on a glass slide (red). (f) Representative the scattering spectra of the complex Au/Ag NRs (black), Au/Ag-GOx NRs before (red) and after (blue) adding $3 \times 10^{-4} \mathrm{M}$ glucose for $60 \mathrm{~min}$.

was separated into two stages. First, the PRS spectra peak of the nanorods gradually red-shifted upon the addition of glucose; it then stoped increasing, and became steady to a saturation value after about $1 \mathrm{~h}$. We also found that the higher was the glucose concentration, the faster did the reaction rate become.

The morphology of the particles was characterized by TEM. After being treated by $3 \times 10^{-4} \mathrm{M}$ glucose for 20 and $60 \mathrm{~min}$, a noticeable collapse appeared on the side of the nanorods, as shown in Fig. 3b. The TEM images indicate that some ultrafine particles scattered around the nanorods, which was caused by forming and loosing of the silver oxide on the NRs' shell after the complex NRs were incubated with glucose. Once the solution encountered disturbances, the outer shell would collapse and the outlines of the NRs will changed (Fig. 3b inset), indicating that $\mathrm{H}_{2} \mathrm{O}_{2}$ could act as a mild etchant to dissolve Ag from bimetallic nanostructures.

Control experiments were carried out in order to investigate the specificity for glucose sensing of the system. As shown in Fig. 4, there was almost no scattering peak shift of Au/Ag-GOx NRs for the fructose, lactose, and sucrose at the same concentrations as glucose.

\section{Conclusions}

In summary, the complex Au/Ag-GOx bimetallic NRs was synthesized, and showed good enzymatic activity. It can thus be exploited as a new method for in situ real-time sensitive optical glucose sensing. By taking advantage of the etching effect of $\mathrm{H}_{2} \mathrm{O}_{2}$ generated from the enzymatic oxidation of glucose, glucose can determine the rate of dissolution of silver so as to adjust the shell thickness of Au NRs, which can be used to tailor the plasmonic response of the nanoparticle sensors. The analytical method based on the biological sensing of single plasmonic nanoparticles is more simple and sensitive than colloidal plasmonic Au/Ag-GOx NR nanocomplexes, which tend to aggregate and require more centrifugation steps. The enzyme-responsive nanocomplex system that we developed here is promising, and could be used as a new analytical platform for the single-particle tracking of $\mathrm{H}_{2} \mathrm{O}_{2}$ or glucose in live cells. 

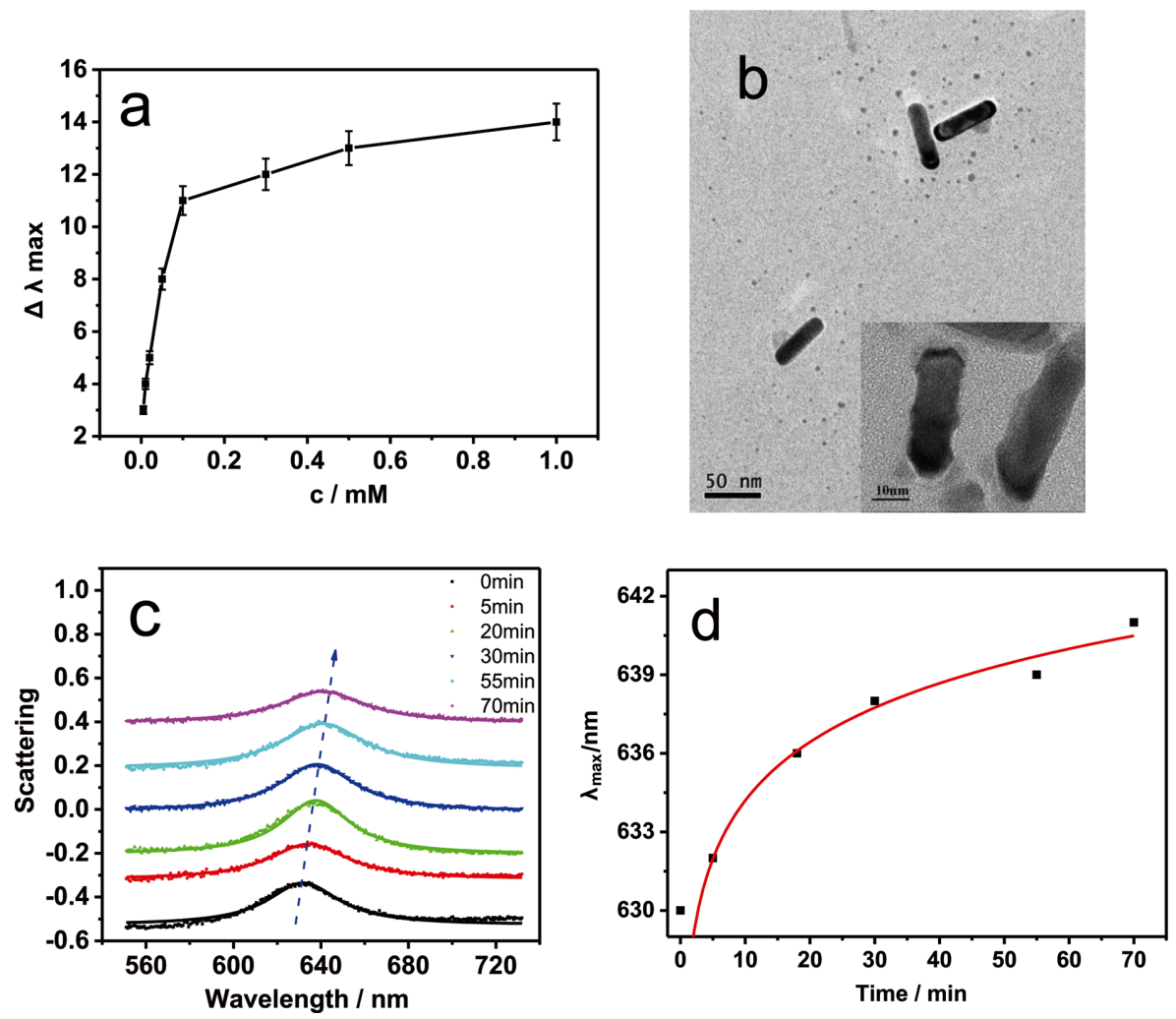

Fig. 3 a) Plot of the scattering peak shift $\left(\Delta \lambda_{\max }\right)$ versus the concentration of glucose for the $\mathrm{Au} / \mathrm{Ag}$ GOx NRs. b) TEM image after the reaction with glucose $\left(3 \times 10^{-4} \mathrm{M}\right)$ for $20 \mathrm{~min}$ (inset) and $60 \mathrm{~min}$. c) Representative time-dependent scattering spectra shift of the complex Au/Ag-GOx NRs upon incubating with glucose $\left(3 \times 10^{-4} \mathrm{M}\right)$, showing the red-shift of the $\lambda_{\max }$, along the direction of the arrow: $0,5,20,30,55$, and 70 min. d) Plot of the scattering peak shift $\left(\Delta \lambda_{\max }\right)$ versus time.

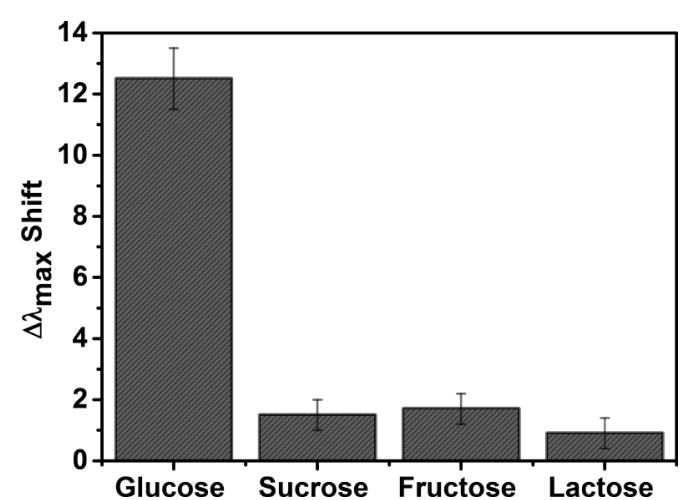

Fig. 4 Scattering spectra shift of the Ag/Au-GOx NRs after $30 \mathrm{~min}$ of incubation with glucose, fructose, lactose, and sucrose with the same concentration $\left(3 \times 10^{-4} \mathrm{M}\right)$, respectively.

\section{Acknowledgements}

This work was supported by the grants from the National Natural Science Foundation of China (21035002) and Natural Science Foundation of Jiangsu Province (project No. BK20130603).

\section{References}

1. J. N. Anker, W. P. Hall, O. Lyandres, N. C. Shah, J. Zhao, and R. P. Van Duyne, Nat. Mater., 2008, 7, 442.

2. P. K. Jain, X. Huang, I. H. El-Sayed, and M. A. El-Sayed, Acc. Chem. Res., 2008, 41, 1578.

3. C. Langhammer, I. Zoric, and B. Kasemo, Nano Lett., 2007, 7, 3122.

4. X. Zhang, Y. L. Chen, R.-S. Liu, and D. P. Tsai, Rep. Prog. Phys., 2013, 76, 46401.

5. S. R. Beeram and F. P. Zamborini, ACS Nano, 2010, 4, 3633.

6. A. J. Haes, S. L. Zou, G. C. Schatz, and R. P. Van Duyne, J. Phys. Chem. B, 2004, 108, 6961.

7. C. L. Haynes and R. P. Van Duyne, J. Phys. Chem. B, 2001, 105, 5599.

8. T. Tan, C. Tian, Z. Ren, J. Yang, Y. Chen, L. Sun, Z. Li, A. Wu, J. Yin, and H. Fu, Phys. Chem. Chem. Phys., 2013, 15, 21034.

9. J. Cao, T. Sun, and K. T. V. Grattan, Sens. Actuators, B, 2014, 195, 332.

10. I. Mannelli and M. P. Marco, Anal. Bioanal. Chem., 2010, 398, 2451.

11. C.-A. Peng and S. Pachpinde, Nanomater. Nanotechnol., 2014, 4.

12. H. He, X. Xu, H. Wu, and Y. Jin, Adv. Mater, 2012, 24 , 1736.

13. H. He, X. Xu, H. Wu, Y. Zhai, and Y. Jin, Anal. Chem., 2013, 85, 4546.

14. I. Willner, B. Basnar, and B. Willner, Febs J., 2007, 274, 302.

15. J. Tashkhourian, M. R. Hormozi-Nezhad, J. Khodaveisi, and R. Dashti, Sens. Actuators, B, 2011, 158, 185. 
16. F. Wang, X. Liu, C.-H. Lu, and I. Willner, ACS Nano, 2013, 7, 7278 .

17. I. Willner, R. Baron, and B. Willner, Biosens. Bioelectron., 2007, 22, 1841.

18. Y. Xia, J. Ye, K. Tan, J. Wang, and G. Yang, Anal. Chem., 2013, 85, 6241.

19. W. Cao, T. Huang, X.-H. N. Xu, and H. E. Elsayed-Ali, J. Appl. Phys., 2011, 109, 34310.

20. M. Liu, J. Chao, S. Deng, K. Wang, K. Li, and C. Fan, Colloids. Surf., B, 2014, 124, 111.

21. L. J. Sherry, R. Jin, C. A. Mirkin, G. C. Schatz, and R. P. Van Duyne, Nano Lett., 2006, 6, 2060.

22. K. Wongravee, T. Parnklang, P. Pienpinijtham, C. Lertvachirapaiboon, Y. Ozaki, C. Thammacharoen, and S. Ekgasit, Phys. Chem. Chem. Phys., 2013, 15, 4183.

23. L. Wang, F. Wang, L. Shang, C. Zhu, W. Ren, and S. Dong,
Talanta, 2010, 82, 113.

24. T. Parnklang, C. Lertvachirapaiboon, P. Pienpinijtham, K. Wongravee, C. Thammacharoen, and S. Ekgasit, RSC Adv., 2013, 3, 12886.

25. S. Wu, A. W. Schell, M. Lublow, J. Kaiser, T. Aichele, S. Schietinger, F. Polzer, S. Kuhn, X. H. Guo, O. Benson, M. Ballauff, and Y. Lu, Colloid Polym. Sci., 2013, 291, 585.

26. H.-X. Han, X. Tian, X.-J. Kong, R.-Q. Yu, and X. Chu, Anal. Methods, 2015, 7, 7989.

27. X. Zhu, L. Sun, Y. Chen, Z. Ye, Z. Shen, and G. Li, Biosens. Bioelectron., 2013, 47, 32.

28. A.-L. Hu, Y.-H. Liu, H.-H. Deng, G.-L. Hong, A.-L. Liu, X.-H. Lin, X.-H. Xia, and W. Chen, Biosens. Bioelectron., 2014, 61, 374.

29. S. A. Khan, G. T. Smith, F. Seo, and A. K. Ellerbee, Biosens. Bioelectron., 2015, 64, 30. 